\title{
El impacto y la reelaboración de las ciencias económico- sociales de América Latina- Caribe en Sri Lanka: Estudio de 3 autores: Gamani Corea, Satchi Ponnambalam y Susantha Goonatilake $\mathrm{e}^{\mathrm{l}}$
}

Eduardo Devés-Valdés*

Resumen. Las ciencias económico-sociales de los 1960s y 1970s han constituido la producción eidética de América Latina-Caribe más reconocida en el mundo. La difusión de éstas fue amplia en diversas regiones, e incluso en lugares insospechados. Entre los numerosos países de África y Asia donde fueron reconocidas debe considerarse Sri Lanka. Teniendo en cuenta el impacto que las ciencias económico-sociales cepalino-dependentistas tuvieron en Sri-Lanka, se estudia la obra de 3 autores, Gamani Corea, Susantha Goonatilake y Satchi Ponnambalam. Se intenta responder, en lo fundamental, a 2 preguntas: ¿Qué aspectos de estas ciencias económico sociales fueron los más recepcionados? Concluyéndose que: en cuanto a los autores más citados se encuentran Raúl Prebisch y André G. Frank; en cuanto a los idiomas, que claramente se recibió más la producción que se encontraba en inglés; y en cuanto al filtro, que recibieron las ideas que había filtrado previamente la comunidad intelectual anglosajona, dedicada a los

\footnotetext{
* Investigador del Instituto de Estudios Avanzados (IDEA) de la Universidad de Santiago de Chile, Román Díaz 89, Providencia, Santiago, Chile. Tel/fax 56-27181360, eduardo.deves@usach.cl Todos los textos citados que aparecen en la bibliografía en otros idiomas han sido traducidos por el autor.
} 
El impacto y la reelaboración de las ciencias económico-sociales...

estudios sobre el desarrollo. ¿Qué elaboraciones teóricas realizaron los srilankeses teniendo a estas ciencias-económico-sociales como insumos? Concluyéndose en dos niveles: el primero, referido a los cruzamientos realizados y allí se detectan tendencias provenientes de la economía del desarrollo, del tercermundismo, del socialismo africano; el segundo, referido a la manera de caracterizar (clasificar) los productos o nuevos sistemas eidéticos aparecidos y en este caso apuntar hacia lo que puede denominarse "teorías del desarrollo tercermundistas e identitarias". Palabras clave: Ciencias económico-sociales. Sri-Lanka. CEPAL. Desarrollo. Dependencia. Circulación de las ideas. Estudios Eidéticos.

\section{Introducción y planteamiento del problema}

a- El objetivo de este trabajo no es realizar un panorama de las ciencias económico sociales en Sri-Lanka ni siquiera durante el periodo estudiado, sino detectar cuales fueron las recepciones de las ideas económico-sociales originarias de América Latina-Caribe (ALC) en algunos autores de ese medioambiente intelectual y, particularmente, determinar como reelaboraron dichas ideas, combinándolas con otras de diversas procedencias.

Es ya algo sabido que, las ciencias económico-sociales elaboradas en ALC entre 1950 y 1970 gozaron de vastas proyecciones hacia muchos lugares del mundo, permitiendo que la región se transformara en importante exportadora de ideas. Es sabido que comunidades intelectuales de numerosos países de Asia y África como de Europa (ver DEVÉS; ROSS 2009; DEVÉS 2010) acogieron las ideas provenientes de ALC, reprocesándolas en hibridaciones varias con elementos eidéticos de otros lugares. Podría trabajarse mucho más acerca de la pregunta por los modos como llegaron estas ciencias ceconómico-sociales a los autores srilankeses, pero es fácil, al menos en una primera aproximación biográfica, darse cuenta que éstas ideas fueron recepcionadas por los srilankeses a través de los contactos con los especialistas en desarrollo que visitaron el país o con los especialistas que encontraron en los organismos internacionales y en las universidades del primer mundo, donde se manejaban tales ideas y donde estudiaron y/o hicieron estadías de investigación, como el Institute of Development Studies de la Universidad de Sussex. 


\section{Eduardo Devés-Valdés}

Numerosas personas interesadas por la cuestión del desarrollo procedentes del Subcontinente Indio y desde Siri-Lanka (S-L) acudieron a universidades extranjeras o participaron de organismos internacionales o redes intelectuales donde las ideas de ALC se encontraban disponibles. Es relevante destacar que hubo igualmente autores de la India, Pakistán y Bangla Desh (DESAI, 1973; DEVÉS, 2009) que también incorporaron en su bibliografía obras de factura en ALC y que ello no sólo ocurrió con la gente que se desempeñaba en las ciencias económico-sociales. La teología internacional también se hizo eco de la producción teológica de ALC y por allí conectó con la producción pedagógica y de las ciencias económico-sociales. Por ejemplo, el teólogo católico srilankés, Aloysius Pieris ${ }^{2}$ ha citado a F. H. Cardoso, A. G. Frank y C. Furtado, como referencias para la discusión (1988, p. 69).

b- En el marco de la interrogación por la circulación de las ideas en los espacios Sur-Sur, es que se formulan las preguntas acerca de las condiciones de recepción y de reelaboración de las ciencias económico-sociales de ALC en S-L: ¿Qué aspectos de estas ciencias económico-sociales fueron los más recepcionados? ¿Qué elaboraciones teóricas realizaron los srilankeses teniendo a estas ciencias económico-sociales como insumos?

Estas preguntas intentarán ser respondidas en el marco de los Estudios Eidéticos como disciplina, focalizándose particularmente en los procesos de reelaboración realizados por los autores de S-L. La reelaboración será estudiada sobre la base de: elementos recepcionados desde ALC; elementos recepcionados desde otras regiones periféricas; elementos incorporados desde S-L y otros elementos; además, teniendo en cuenta el tipo de cruzamientos o combinaciones realizados entre tales elementos.

c-Podría sonar algo trivial reducir el impacto, la recepción y reelaboración a 3 autores, aunque ciertamente no lo sería si estos fueran botones de muestra de un fenómeno mayor. Sin embargo, no está nada claro que sea así. Los autores tratados están entre los poquísimos que, en la pequeña comunidad intelectual de S-L, se hicieron eco de los planteamientos procedentes de ALC.

Este trabajo adquiere validez no tanto por dar a conocer una importante tendencia de pensamiento en S-L, puesto que en verdad 
El impacto y la reelaboración de las ciencias económico-sociales...

no lo fue, sino que por la intención de mostrar y hacer consciente a una intelectualidad, como la de ALC, lo que ha sido la proyección de su propio quehacer. Nuestra intelectualidad desconoce, casi completamente, las proyecciones de su pensamiento más allá de las propias fronteras. Mostrar casos de recepción y reelaboración en diversos lugares de África y de Asia, como se ha ido haciendo, adquiere entonces importante significación.

Aparte de informarnos sobre las proyecciones de nuestro pensamiento, el reconocimiento de estos casos tiene significación también por la excepcionalidad de estos experimentos eidéticos, en los cuales las ideas originarias de ALC han sido cruzadas con ideas originarias de África o Asia e incluso con otras ideas, también procedentes de nuestra región, particularmente del Caribe, en cruzamientos que curiosamente no se hicieron acá mismo. Por otra parte, más allá de los estudios acerca de la recepción, la proyección o la circulación de las ideas, estos estudios tienen algo de fascinante: darse cuenta de los insospechados caminos, mutaciones, cruzamientos y productos que tuvieron su origen en el medio cepalinodependentista, cosa que esta comunidad ni siquiera soñó.

\section{La obra de tres autores de Sri Lanka que acogieron las ideas provenientes de ALC}

\section{La obra de Gamani Corea}

Nacido en 1925, estudió en Royal College, Colombo; en la Universidad de Ceilán (antiguo nombre de S-L), luego en el Nuffield College de Oxford y en el Corpus Christi College de Cambridge. Fue Secretario General de la UNCTAD (United Nations Conference on Trade and Development). Entre otras actividades académicas fue Honorary Fellow, del Institute of Development Studies, de la Universidad de Sussex.

En sus Memorias, Corea (2008) recuerda los contactos con varios de los más importantes economistas del desarrollo. Sabemos que varias de ellas estaban familiarizadas con algunas de las ideas 


\section{Eduardo Devés-Valdés}

cultivadas en ALC, particularmente con las tesis de R. Prebisch acerca del deterioro en los términos del intercambio o de la categoría centro-periferia y con las de Arthur Lewis, sobre industrialización por invitación, sobre la abundancia de mano de obra y sobre la coexistencia en un país de un sector moderno y otro tradicional. Es el caso de Prasanta Chandra Mahalanobis, de Nicolás Kaldor, J. K. Galbraith, Oscar Lange, John y Ursula Hicks y Gunnar Myrdal.

Gamani fue uno de los herederos del pensamiento de Raúl Prebisch, aunque no tanto del cepalismo clásico de los 1950s, como teoría del desarrollo hacia adentro, sino de la etapa en que Prebisch teorizó acerca de un comercio justo, en la época en que pensó como un intelectual orgánico del Tercer Mundo o del Sur, es decir del Prebisch puesto claramente en el escenario mundial, en la UNCTAD, y ya no en la asesoría a los estados de ALC. Prebisch describió lo que él mismo llamó la "cuarta etapa de mi pensamiento", en relación con la UNCTAD ${ }^{3}$ y ubicada entre 1963 y fines de esa década, como orientada hacia los problemas de la cooperación internacional. Este pensamiento se expresó presentando un conjunto de recomendaciones que, según señaló, se afirmaron en lo que había pensado anteriormente en el marco de la CEPAL (1983; 1986). Estas recomendaciones sobre política económica, constituyeron el punto de partida para el trabajo entre los gobiernos miembros. Aquí se inició el diálogo Norte-Sur, aunque más que un diálogo fue una serie de monólogos paralelos, monólogos que no conducían a la acción concreta sobre los problemas más fundamentales de la cooperación internacional $(1983 ; 1986)$. Es en esta línea temática e inspirado en el pensamiento de este Prebisch, donde se encuentra Gamani Corea.

Corea conoció personalmente al Prebisch de la UNCTAD, cuyo pensamiento, aunque según confesión de Prebisch no había evolucionado mucho, se veía enfrentado a otro escenario y marcando otros énfasis. Corea ha recordado que cuando conoció a Prebisch, poseía un background en economía, pero los temas que estaba elaborando Prebisch eran relativamente nuevos para él, de modo que le dieron una nueva dimensión a su pensamiento y a la tarea que se proponía (COREA, 2001). En su libro Need for Change. Towards the New Internacional Order (Necesidad de cambio. Hacia el Nuevo Orden Internacional) de 1981, citó varias veces a Prebisch 
El impacto y la reelaboración de las ciencias económico-sociales...

señalando las cuestiones que éste planteó en el momento inicial de la UNCTAD, de lo cual él sería el continuador. Señaló que la UNCTAD, siguiendo el liderazgo de Prebisch, buscó atender a la inadecuación que significaba el orden económico internacional, como soporte para el desarrollo del Tercer Mundo (1981, p. 1). Uno de los temas principales de Prebisch en ese esquema fue la necesidad de una nueva política comercial $(1981$, p. 2), precisamente porque la existente no era conducente hacia el desarrollo.

\section{La obra de Satchi Ponnambalam}

El abogado, economista y activista político, Satchi Ponnambalam elaboró una interpretación de la historia de S-L, inspirándose en el pensamiento dependentista combinado con otras ideas emergidas en el mundo periférico. Publicó en 1981 su libro Dependent capitalism in crisis. The Sri Lankan economy 1948-1980, (Capitalismo dependiente en crisis. La economía srilankesa) en el cual se proponía, entre otras cosas, dar cuenta de la herencia recibida del sistema colonial y que consistía en "un sistema económico subdesarrollado en la periferia, dependiente del centro y dominado por una clase orientada hacia el extranjero" (1981, p. 1). Este sistema no correspondía, según su visión de las cosas, a un estado infantil o temprano, como los que habían existido en los países desarrollados en la época pre-capitalista, y que fuera capaz de un completo desarrollo. Se trataba, en cambio, de una estructura completamente desequilibrada y deformada. Según él, ello se expresaba, entre otras cosas, en que el sistema de plantación introducido había permanecido divorciado de la economía agraria tradicional y en que las clases dominantes estaban orientadas hacia el consumo externo. El establecimiento de una economía de plantación, durante la era colonial, dividió la economía nacional entre un sector moderno y otro tradicional, creando así un país con una economía dual (1981, p. 173).

En este esquema, entendía el rol de la economía nacional, en la periferia del sistema capitalista mundial, como exportadora de materias primas y/o importadora de bienes de consumo de lujo. 


\section{Eduardo Devés-Valdés}

Este carácter, que provenía de los últimos años del período colonial, quiso ser modificado a través de una política de sustitución de importaciones, pero ello no tuvo los resultados deseados, pues la sustitución no fue capaz de satisfacer las necesidades del pueblo y las industrias de sustitución de importaciones fueron dependientes de la importación de materias primas y de la importación de tecnología (1981, p. 13). En el momento de la independencia existía una estructura de clases que "aunque las antiguas distinciones de raza y casta continuaban siendo fundamentales" se había modificado de acuerdo a la nueva inserción en la economía internacional (1981, p. 15). Había sectores de la clase alta que no tenía nada en común con la gente común, en muchos sentidos se había hecho británicos y querían permanecer como tales, habían sido educados en inglés, se habían convertido al cristianismo, habían desarrollado cierto grado de espíritu empresarial, habían adquirido algunas de las destrezas de los antiguos amos y repudiaban los valores y costumbres ancestrales (1981, p. 16).

\section{La obra de Susantha Goonatilake}

Se formó inicialmente como ingeniero eléctrico para luego dedicarse a la sociología, disciplina que estudió en S-L y en Gran Bretaña. En este afán se volcó posteriormente a los estudios acerca del desarrollo insertándose en el Institute for Development Studies, Universidad de Sussex. Comenzó a cuestionar la validez del discurso sobre el desarrollo elaborado en Occidente, conociendo allí la obra del los autores de ALC (especial agradecimiento hace a Osvaldo Sunkel por sus aportes) $)^{4}$, dio los primeros pasos en su trabajo acerca de los aportes no-occidentales al saber sobre el desarrollo, lo que se prolongó hacia el discurso de las ciencias y humanidades y hacia el conocimiento en general.

Durante su permanencia en el IDS-Sussex, se ocupó tempranamente del impacto del pensamiento occidental sobre las regiones periféricas y acerca de cómo capitalizar o elaborar un pensamiento propio. En el IDS-Bulletin publicó sendos trabajos acerca de estos 
El impacto y la reelaboración de las ciencias económico-sociales...

asuntos, asumiendo la importancia de las innovaciones realizadas a este respecto por autores de ALC. En dichos trabajos, citó a O. Sunkel, C. Furtado, A. G. Frank, Th. dos Santos, R. Stavenhagen, así como a D. Seers y otros autores que se manejaban con la conceptualización cepalino-dependentista, ocupándose específicamente del problema del colonialismo y la dependencia intelectual. En su discurso se advierten claramente las herencias de Rodolfo Stavenhagen (1971) que apuntó a la "descolonización de la academia". Goonatilake afirmó que existía, internacionalmente, una comunidad académica que funciona según el modelo centro-periferia, o un modelo burocrático, que consideraba que el conocimiento serio sólo sería producido en el centro. La nueva relación en la academia, podía ser mejor descrita, en términos generales, citando y parafraseando aquello que ha dicho Sunkel sobre las multinacionales (1975a, p. 8-9). Dicho de manera más radical, "en el caso de los países periféricos dependientes, el conocimiento legitimado es determinado por las particulares relaciones socio-económicas entre el centro y a periferia dependiente" (1975b, p. 34). Y se preguntaba, en este contexto, por qué ciertos países del Tercer Mundo no han producido "un A. G. Frank, un Th. Dos Santos, un R. Stavenhagen o un C. Furtado" y "por qué los latinoamericanos han sido capaces de producir un vigoroso cuerpo de conocimiento sobre el desarrollo (el cual está siendo legitimado y re-transmitido por centros como el IDS hacia el resto del Tercer Mundo)", para luego responderse, "porque en los 1960s ellos, críticamente, confrontaron los análisis y soluciones propagados por el centro metropolitano" (1975b, p. 34). Y si hay excepciones en Sri Lanka, como es el caso de Gamani Corea, se debe a que "ha seguido las posiciones creadas inicialmente por los latinoamericanos" y, en todo caso, no le parece que el trabajo de éste o de otros srilankeses sea una contribución del nivel de la aportada por los latinoamericanos (1975b, p. 34). Es precisamente en el marco de estas premisas que deben entenderse sus trabajos posteriores apuntando hacia una agenda para la intelectualidad del Sur. 


\section{Algunos componentes del medioambiente intelectual de Sri Lanka}

Se han diseñado algunos trazos del pensamiento de los tres autores seleccionados durante el período en estudio. Procede ahora presentar algunos trazos de la trayectoria eidética de la sociedad en que se desenvolvieron y para la cual escribieron, aunque su pensamiento, particularmente el de Gamani Corea, no haya pretendido realizarse de manera local sino que globalmente. Por otra parte, es muy obvio, que autores internacionalizados como los que se estudian, en ningún caso se limitaron a un ecosistema eidético restringido en términos nacionales, sino que acogieron elementos en sus viajes, estadías en el extranjero, y por cierto, en sus lecturas disponibles en la biblioteca universal. En tal sentido, más relevante que retratar el medioambiente intelectual de Colombo en las décadas en las cuales se formaron los tres autores y poco antes, es interesante poner en relieve las ideas disponibles en ese medioambiente. Gamani Corea se ha referido insistentemente a las ideas que recibió de Prebisch, Ponnambalam leyó a F. Fanon y a J. Nyerere y Goonatilake leyó y dialogó, por ejemplo, con los latinoamericanos en Sussex.

En la trayectoria eidética de Ceilán-S-L, especialmente en el medioambiente intelectual de Colombo y las principales ciudades del país, pueden destacarse varios elementos que se expresaron entre fines del siglo XIX y 1950, aproximadamente. Se trata apenas de mencionar las tendencias más significativas para el objetivo que interesa: señalar los cruzamientos eidéticos que más tarde se producirían con las ideas económico-sociales procedentes de ALC.

1-El pensamiento budista-revivalista, que tuvo un desarrollo importante luego de la "provocación” teosófica de la década de 1870, fue elaborado particularmente por Anagarika Dharmapala, realizándose como la primera versión del identitarismo srilankés (o sinhalés) (ROBERTS, 1997). El pensamiento de Anagarika Dharmapala, en una de sus líneas, se orienta a combatir la idea de superioridad británica y occidental, para lo cual se remonta a tiempos remotos para comparar la propia civilización con la relativa barbarie sajona de la época. Esta afirmación de lo propio se orienta a defender la 
El impacto y la reelaboración de las ciencias económico-sociales...

sobrevivencia de su cultura, entendida principalmente con las enseñanzas del budismo, ante la amenaza colonial.

2-El movimiento teosófico, por su parte, emprendió la sistemática fundación de escuelas, dejando una impronta duradera en la cultura del país, en una dialéctica que implantaba sistemas educativos occidentales, exaltando lo oriental. Ello conectó más tarde al movimiento teosófico con el nacionalismo indio, también asociado a la teosofía, particularmente estando Annie Besant a la cabeza del Indian National Congress.

3- Hacia 1900 se encontraban también disponibles en CeilánS-L las ideas del nacionalismo indio, en su trayectoria hindú y en su trayectoria islámica. Se destacó entonces un pensamiento derivado del revivalista hindú, con referencias del pensamiento nacionalista procedente de Kolkata, principalmente desde 1900 en adelante. Por esa misma época se desarrolló el pensamiento revivalista islámico inspirado en el movimiento de Hyderabad (actual Pakistán) que, desde 1860, se venía gestando en dos líneas bifurcadas, una más identitaria (Deoband) y otra más centralitaria (Aligarh).

4- El nacionalismo srilankés, si así puede llamarse, se abrevó parcialmente en los nacionalismos indios, hindú y musulmán, aunque pronto adquirió un discurso propio, particularmente en su versión sinhala, pues la versión tamíl fue más tardía. Este pensamiento nacionalista tomaría mayor fuerza, como en todo el Subcontinente Indio, una vez que M. Gandhi comenzara a ejercer su liderazgo. A partir de la confluencia entre identitarismo, teosofía y nacionalismo, derivó un "dharmapalitismo" gandhista-nacionalista-sindicalista, desde la segunda década del siglo XX y luego un "dharmapalismo" antiimperialista, desde mediados del siglo, con elementos de marxismo y nacionalismo, en especial entre monjes budistas.

5-El pensamiento de corte socialista (JUPP, 1978), en versiones materialistas, se hizo presente en los 1930s. El Partido Sociedad Igualitaria fue fundado en 1935 por personas educadas en Gran Bretaña, algunas de las cuales discípulas de Harold Laski y de inspiración fabiana. El partido abogaba por la independencia nacional y por un socialismo evolutivo. Es de resaltar, por otra parte, la importante trayectoria del pensamiento trotskista, siendo S-L en términos relativos uno de los lugares más significativos del mundo para este movimiento eidético y político (LERSKI, 1968, p. vii ss). 


\section{Eduardo Devés-Valdés}

6-Luego de la II Guerra Mundial y en los primeros años de vida independiente, el pensamiento nacionalista dio un salto político y social, aunque también eidético, con la versión nacionalista y socialista bandaranaike. Aquí maduró lo que se llamó "socialismo budista", donde confluyeron elementos social-demócratas, incluso en las versiones "neo-fabianas" del desarrollismo, con reivindicaciones budistas e idiomáticas e incluso "comunalistas", de defensa de la comunidad sinhala (JUPP, 1978, p. 631).

7-Hacia 1950, se instalaron las ciencias económico-sociales con su paradigma de la modernización y el desarrollo (ASIRWATHAN, 1975), de la mano de las misiones de funcionarios y académicos internacionales, contratados para prestar asesoría a un estado en vías de constitución independiente. En el pensamiento de estos especialistas convergían la propuesta del desarrollo y el socialismo evolutivo, asociado a la idea del estado-bienestar. En los 1950s y 1960s importantes figuras de las redes de la economía del desarrollo se hicieron presentes en S-L, articulándose a la política local.

8-Se desarrolló, al interior del campo de las ciencias sociales, como antes en el pensamiento sobre asuntos políticos y culturales, una línea de pensamiento fuertemente identitaria que rechazaba los modelos epistémicos occidentales como negativos para el propio quehacer, por inhibir un pensamiento propio. En el trabajo "Las ciencias sociales en Sri Lanka: política, investigación y formación", publicado en 1975, R. S. Asirwathan señala que "los hombres de ciencia asiáticos trabajan sobre problemas y en sectores de investigación determinados en Occidente, aplicando métodos y teorías occidentales. Para permitir a los sociólogos de Asia resolver ellos mismos sus problemas y construir sus propias teorías adaptadas a su sociedad, sería necesario que los países de Asia pusieran en marcha un programa de colaboración regional". En todo caso, las ciencias sociales, sostiene, no pueden desarrollarse eficazmente sino en la perspectiva de problemas locales, por lo que es necesario que la incorporación de ciertos aspectos pertinentes de la teoría social occidental en la enseñanza de universitaria de los países de Asia se haga con discernimiento. Ello, porque es difícil para un universitario medio percibir las aspiraciones del pueblo, encontrándose en una situación económica y cultural diferente (1975, p. 291). Para 
El impacto y la reelaboración de las ciencias económico-sociales...

reforzar sus ideas Asirwathan cita al Dr. Ralph Pieris, ex miembro de la Universidad de Ceilán, quien consideró "que la investigación extranjera es perjudicial para el desarrollo nacional de las ciencias sociales" (apud ASIRWATHAN, 1975, p. 291).

\section{Reelaboraciones y cruzamientos eidéticos por parte de los srilankeses, teniendo en cuenta los insumos provenientes de ALC.}

Una primera lectura, algo apresurada o focalizada en pocos textos, podría llevar a creer que las líneas de pensamiento recién reseñadas, salvo las ciencias económico-sociales del desarrollo, recién llegadas al país, casi no tienen presencia en los autores que se estudian y ello no sería demasiado sorprendente. Por casi todas partes en el Tercer Mundo, las ciencias económico-sociales se instalaron hacia 1950 como un producto de importación, estableciendo conexiones con el medioambiente intelectual de las sociedades pero entroncándose escasamente en la trayectoria eidética de estas. Sin embargo, lecturas hechas con mayor perspectiva, permiten detectar, en los autores ${ }^{5}$, diversos componentes de escuelas o tendencias de pensamiento, presentes en el medioambiente intelectual de forma previa a la llegada de las ciencias económico-sociales. Esto llevó, particularmente a Goonatilake, a realizar cruzamientos eidéticos novedosos, como por ejemplo, combinar el identitarismo dharmapaliano, el dependentismo y las teorías sobre la historia de la ciencia de Thomas Kuhn.

\section{Las reelaboraciones de Gamani Corea}

Gamani Corea más que reelaborar el pensamiento procedente de ALC, particularmente prebischiano, realizando cruzamientos con otras escuelas de pensamiento, procedentes de África o Asia, como ocurrió con Ponnambalam o Goonatilake, desarrolló y concluyó a partir de la propia dinámica de las ideas de Prebisch, combinándolas 


\section{Eduardo Devés-Valdés}

con otras que se manejan en el ámbito de los organismos internacionales. Manteniéndose en un mismo ámbito de preocupaciones, maduró un pensamiento y lo fue interpelando con los cambios a nivel mundial y con las nuevas elaboraciones que se generaban en el ámbito de los organismos internacionales para el desarrollo, pero no asumió otras líneas de pensamiento y, en consecuencia, no recurrió a tendencias procedentes de su propio país ni de regiones del mundo periférico.

En 1984, Corea sostenía que existían bases teóricas en el pensamiento económico para la intervención en el comercio acerca de las materias primas. La teoría económica clásica ha dado algunas bendiciones al concepto de acción gubernamental para regular los mercados de materias primas. Esta idea de intervención no ha venido, como podría pensarse, desde el ataque socialista al mercado, sino más bien desde el reconocimiento de falta de simetría en la manera que el mercado opera en el campo de la agricultura, en contraste con el área de las manufacturas o dicho de otra manera. Debido a la naturaleza peculiar de las respuestas de la oferta a los precios, en el campo de los productos primarios, el mercado no es percibido como auto-regulado. Esto ha motivado un cierto grado de regulación, no para reemplazar el mercado sino para progresar con él (1984a, p. 167). En ese momento, Corea estaba incorporando en su discurso la idea de "interdependencia", una idea políticamente correcta, que le permitía argumentar sobre la necesidad del desarrollo de los subdesarrollados como base para el bien de todas las economías del mundo. En respuesta a la crisis económica: la recuperación estaba condicionada a una adecuada interdependencia, lo que quería decir una política nacional e internacional concertada. Ello le parecía más racional que continuar debilitando las instancias de diálogo internacional que conducirían al desarrollo, como se estaba haciendo por esos años (1984b, p. 1-2).

Poco después, a fines de los 1980s, Corea se quejaba de que USA no se comprometiera con la cuarta década del desarrollo que debía iniciarse pronto, habiendo sido precisamente un presidente de ese país, J. Kennedy, quien había lanzado la primera década del desarrollo (1990, p. 17). Esta cuarta etapa se iniciaba con pocos auspicios, luego de la década perdida de los 1980s y de la falta de 
El impacto y la reelaboración de las ciencias económico-sociales...

compromiso de USA con esta iniciativa. Pero la debilidad de esta cuarta década era más radical, pues incluso se había perdido el consenso respecto de la necesidad de colaboración con el desarrollo (1990, p. 19).

Corea proyectó numerosos elementos de Prebisch, como la necesidad de distribuir mejor los frutos del desarrollo y la importancia del conocimiento, pero por otra parte se ocupó de temas presentes en el medio internacional como el medioambiente (1990, p. 21) y la mujer en el desarrollo, que no estaban presentes en la agenda (ni en el imaginario) de Prebisch (1990, p. 21). En 2001 señalaba "Yo pienso que los países del Tercer Mundo necesitan instalar en conjunto una nueva agenda, una nueva plataforma que combine, por una parte, aquello que es relevante de la antigua plataforma, que viene de la UNCTAD y, por otra parte, la respuesta a los nuevos problemas desde su punto de vista, no necesariamente rechazando el proteccionismo" (2001). Precisamente, proyectando el planteamiento prebischiano dentro de su propia coherencia, afirmaba que existían una cantidad de problemas que los países en desarrollo deben proponer y que no van a ser propuestos a ellos por los países desarrollados ni por los organismos multilaterales. "Pienso que este es el mayor desafío que enfrentan y puede hacerse echando mano de organismos como la UNCTAD", pues ésta puede hacer una contribución importante. "No hay otra instancia para el Tercer Mundo con tal cohesión para llevar estos asuntos. Los países del Tercer mundo deben actuar de consuno, a pesar de las diferencias existentes y que son mayores que en 1964, cuando se fundó la UNCTAD. Este es el desafío del momento y es el desafío que la UNCTAD puede mejor que cualquier otro organismo ayudar a alcanzar" (2001).

\section{Las reelaboraciones realizadas por Satchi Ponnambalam}

Ponnambalam, a diferencia de Gamani Corea, afirmó su constructo teórico citando una abundante bibliografía, tanto autóctona como externa, que facilita la detección de sus fuentes y de los 


\section{Eduardo Devés-Valdés}

cruzamientos eidéticos que realizó. Sus referencias claves fueron a la escuela de la economía del desarrollo, fundamentalmente a algunos de los clásicos, como Thomas Balogh, N. Kaldor, Gunnar Myrdal y Ragnar Nurkse, otros pertenecientes a la escuela neo-marxista como Paul Baran, Paul Sweezy y Charles Bettelheim, además de T. Szentes y Pierre Jalee. También aludió a V. I. Lenin, del cual citó varios trabajos; a numerosas figuras de la escuela inglesa, que se había inspirado tanto del pensamiento latinoamericano como del neo-marxismo, particularmente a Dudley Seers. Citó igualmente a los dos más importantes economistas de ALC: varios textos de Raúl Prebisch (y también el trabajo de June Flanders sobre él) y Arthur Lewis, y al mas reconocido teórico de la dependencia A. G. Frank. Fueron también frecuentes sus referencias a su compatriota Gamani Corea que, como ya sabemos, había tenido frecuente comercio intelectual con los autores del desarrollo y con los latinoamericanos. Citó, por otra parte, al importante cientista económico-social indio Jagdish Bhagwati, también conocedor de las ciencias económico-sociales de ALC y participante de las redes de cientistas económico-sociales del Tercer Mundo (DESAI, 1973).

Lo más importante, desde el punto de vista de los estudios eidéticos, no desde la economía o la planificación, es la sofisticación del constructo teórico elaborado por Ponnambalam, a partir del variado conjunto de componentes a los que echa mano. Su objetivo fue explicar lo que, según su diagnóstico, estaba ocurriendo económicamente con Sri Lanka y entregar soluciones.

En sus quehaceres de reelaboración de las ideas mediante cruzamientos, Ponnambalam combinó un conjunto más o menos grande de elementos, pudiendo hacerse un paralelo con que ocurrió en Tanzania con Walter Rodney, tanto por la variedad de ingredientes que incorporó ${ }^{6}$ como por la recurrencia a Fanon y a J. Nyerere (LEWIS, 1998; DEVÉS, 2005). Ingredientes iniciales de Ponnambalam fueron Prebisch y el cepalismo, recepciones que no tardó en criticar. En este marco debe entenderse su alusión a A. G. Frank, pues Ponnambalam se hizo eco de las críticas dependentistas al pensamiento cepalino, principalmente aquellas que se referían al hecho que el pensamiento cepalino condujo a políticas que no alcanzaban 
El impacto y la reelaboración de las ciencias económico-sociales...

los propios objetivos planteados y que incluso se tornaban contra éstos, aumentando el subdesarrollo y la dependencia.

Por otra parte, las citaciones de Julius Nyerere (y de autores como J. Maeda y I. Kaduma, que habían expuesto acerca del "socialismo-ujamaa" de Nyerere) como también las citaciones que hace de Kwame Nkrumah y Dany Nabudere muestran la acogida que brindó al pensamiento africano sud-sahariano. Ponnambalam armó su discurso acerca del desarrollo nacional, apoyándose en una visión romántica o populista, que consideraba que el pueblo liberado de la dependencia, invariablemente tomaba las decisiones correctas, recogió las ideas de Julius Nyerere (a quien define como "un pragmático y realista planificador social", siguiendo su afirmación que los países del Tercer Mundo necesitan: tierra, trabajo, buenos líderes y buenas políticas y que las dos primeras cosas existían, en tanto que las dos últimas $\left.\mathrm{n}^{\circ}, 1981,182\right)$, aunque no tomó su idea más específica, la del socialismo-ujamaa, acudiendo particularmente a la cuestión de la self-reliance y otros elementos y particularmente aquellos referidos a un desarrollo social, autóctono, identitario y humanista, que enfatizaba cuestiones como llegar a ser dueños del propio destino (1981, p. 184).

Por otra parte todavía, acogió las ideas de Frantz Fanon para realizar una interpretación del colonialismo, entendiéndolo como una aceptación del colonizado de la dominación, por una suerte de complejo de inferioridad, debería superarse afirmando la selfreliance, particularmente en su interpretación nyeyeriana. Es decir, intentó una interpretación de la realidad social o psico-social de Sri Lanka, que no se basaba en las ideas de Max Weber, cosa que podría haber sido más o menos convencional entre quienes se ocupan del desarrollo, sino que afirmándose en las ideas de Fanon. Según el análisis de lo social que realizó Ponnambalam, la época colonial dividió la sociedad no sólo en dos clases sino verdaderamente en dos naciones, los ricos y los pobres. El sistema de castas, cimentado en el llamado alto y bajo nacimiento y santificado por la religión, era aceptado como inmutable. Así, la estructura social fue construida sobre la base de desigualdades, privilegios de clase, opresión, explotación y discriminación. La sociedad aceptó la desigualdad y la resultante degradación de la personalidad humana. Las vastas masas 


\section{Eduardo Devés-Valdés}

el pueblo, en consecuencia, carecen de self-reliance. Esa fue la base sobre la cual se fundó el colonialismo. La conquista colonial hizo que el pueblo colonizado aceptara un estatus de segunda categoría en su propio país. Sólo la lucha de masas permitiría al colonizado reconquistar su auto confianza (1981, p. 177).

Dicho de otra manera, asumiendo parcialmente las propuestas provenientes de ALC, las reformula inspirándose en la obra de Julius Nyerere, interpretándolo "fanonianamente". Su propuesta se afirmó en la self-reliance, entendida como "autosuficiencia considerando las necesidades básicas del pueblo en alimentación, habitación, salud y educación", cosas que no podrían obtenerse dependiendo del mundo exterior. Aunque, por otra parte, no debía tampoco entenderse la self-reliance como "aislamiento", sino inspirándose en la convicción que "el desarrollo debía venir desde dentro y ser fundado en los propios recursos nacionales, naturales y humanos, de acuerdo al ethos del pueblo" (1981, p. 186), explicitado esto en su idea del desarrollo como "crecimiento económico + cambio social" (1981, p. 183). De este modo, sintetizó que "la prioridad más importante para todos los países subdesarrollados era la transformación económica desde la dependencia hacia la self-reliance, dejando de funcionar como apéndices del sistema capitalista internacional, para poner la confianza en los recursos domésticos, la tierra y la gente, con el fin de producir para las necesidades de la población (1981, p. 185).

Ponnambalam se ocupó igualmente de la historia del pueblo tamil en S-L y de estudiar los modos como ese pueblo, al cual él mismo pertenecía, estaba siendo devorado, según sus palabras (1984). Es significativo como proyectó algunas de las categorías que manejó para pensar el subdesarrollo de S-L, para pensar entonces la cuestión tamil. Los tamiles eran a la población sinhala, en cierto modo, lo que S-L era al capitalismo internacional. Pero estas categorías a que recurrió para expresar esto no fueron principalmente las cepalino-dependentistas, sino mucho más aquellas que tomó desde Fanon y Nyerere, es decir, no aquellas que aludían a la cuestión económica, sino psico-cultural. 
El impacto y la reelaboración de las ciencias económico-sociales...

\section{Las reelaboraciones de Sunsantha Goonatilake}

Como ya se ha enunciado, Goonatilake se proyectó desde las ciencias sociales y desde el pensamiento sobre el desarrollo, apartándose de estos asuntos, para focalizarse más precisamente en lo que siempre le interesó: la producción de conocimiento en las regiones periféricas (y/o no-occidentales), con el fin de detectar allí gérmenes de creatividad anteriores o diferentes a los de Occidente. En tal sentido, se abrió a un tipo de pensamiento que no se limitaba al ámbito del discurso de las ciencias económico-sociales, como Corea o Ponnambalam.

Goonatilake se ha dedicado posteriormente al estudio de las expresiones eidéticas no occidentales y a los aportes que éstas pueden significar para la humanidad, asociadas a los cambios mundiales, expresión de lo cual ha sido su libro Aborted discovery. Science and creativity in the Third World (Descubrimiento abortado. Ciencia y creatividad en el Tercer Mundo), de 1984, en el cual intentó cartografiar la inhibición del quehacer de la periferia por parte del centro (SANKATSING, 2007, p. 10) y donde llamó a la intelectualidad del Tercer Mundo a desligarse de las tendencias de la ciencia occidental, para retomar su propia creatividad. En otro trabajo, publicado en 1993, "Modern science and the periphery. The characteristics on the dependent knowledge" (La ciencia moderna y la periferia. Las características del conocimiento dependiente), retomando la discusión acerca de los problemas del quehacer científico en la periferia y la dependencia intelectual, insistió en conceptos vertidos en los 1970s, haciendo referencia en la bibliografía a sus textos de esos años, marcando así la continuidad de una reflexión. Asumiendo uno de los más reiterados "motivos periféricos" allegó pruebas para mostrar cuanto tomó la ciencia y la tecnología occidentales (muchas veces sin reconocerlo) de la elaborada en el Sudeste Asiático y de otros lugares diferentes de Occidente.

Ha sido en el marco de este proyecto intelectual, que se ha prolongado largamente en la obra de Goonatilake, en que aparecen citados inicialmente a los autores de ALC, como capaces de crear una escuela de pensamiento original, diferente de aquello que les venía desde el discurso del centro. Aunque de hecho, no tomó sólo 


\section{Eduardo Devés-Valdés}

el ejemplo de los latinoamericanos, que "en los 1960s críticamente confrontaron los análisis y soluciones propagados por el centro metropolitano" (1975b, p. 34), sino que tomó también categorías específicas para entender la realidad: dependencia, centro/periferia, descolonización de las ciencias sociales. Casi 20 años después, en su texto sobre el conocimiento dependiente (1993), continuaba expresándose con nociones como "países periféricos", "países dependientes" y pasando desde allí hacia la noción "conocimiento dependiente".

Por otra parte, ya en 1975, conectaba estas categorías latinoamericanas con las de Thomas Kuhn argumentando que "el conocimiento está, en amplia medida, determinado socialmente" (1975b, p. 34) y asociando esta determinación con la condición dependiente en el marco de las relaciones centro/periferia, aunque los latinoamericanos han sido capaces de "producir un vigoroso cuerpo de conocimiento sobre el desarrollo, que ha sido ahora legitimado y retransmitido por centros como el IDS-Sussex hacia el resto del Tercer Mundo (1975b, p. 34). Los cientistas económico sociales latinoamericanos y la postura de Stavenhagen, de un saber descolonizado, se oponen precisamente a los que en S-L, u otros lugares, siguen actuando a la manera de los "mimic-men" (copiones, imitadores) que retrató Vidia Naipaul (1975b, p. 35),

En el marco de estas reflexiones deben entenderse los entronque de Goonatilake con trayectorias del pensamiento en S-L, revelando algunos de los elementos que ha tomado desde la trayectoria eidética nacional, como es la recuperación de las ideas y la figura del líder budista Mapalagama Vipulasara, a quien caracteriza como "un monje para nuestro tiempo", un "heredero de Dharmapala" y un continuador de una "obra de cultura anticolonial", de "ciencia alternativa", de "pan-budismo", de "defensa de la cultura srilankesa", de "cultura de la paz" (2000, p. 1-2), y el entronque con la línea de pensamiento que se desenvuelve en el seno de las ciencias sociales, que expresa R. S. Asirwathan y que refuerza citando al Dr. Ralph Pieris, en relación a los efectos negativos del saber occidental para el autóctono.

En trabajos posteriores, Goonatilake ha continuado insistiendo en esta línea, afirmando que las ciencias proyectadas desde el 
El impacto y la reelaboración de las ciencias económico-sociales...

centro han dirigido y distorsionado el saber de las periferias (1993, p. 259), o que "el Nuevo siglo estará marcado por varios cambios dramáticos, que dejarán su efecto en el conocimiento, la ciencia y la tecnología. Uno es el cambio en los centros de gravedad del mundo en términos económicos y políticos. Otro conjunto de cambios se produce en el campo de la ciencia y la tecnología, partiendo por el cambio de la tecnología de la información y la biotecnología. Estos cambios tendrán profunda influencia en las relaciones geopolíticas de ciencia y tecnología $(2002$, p. 1). Tres líneas de pensamiento (la cepalino-dependentista, las teorías sobre historia de la ciencia y el identitarismo nacionalista srilankés) se articulan en sus reivindicaciones de un saber autóctono-alternativo, que él entiende algo rusonianamente (de Rousseau) como proveniente de un buen salvaje inquieto e inquisitivo, que logra entender y extraer de la naturaleza mejor de lo que lo pueden hacer las multinacionales farmacéuticas (1993, p. 265-267).

\section{Conclusiones}

La conclusión se focalizará en 3 puntos: si las elaboraciones que llevaron a cabo estos autores tendrían algo en común, constituyendo algo así como una escuela sirilankesa de pensamiento; si sobre la base de elementos procedentes de ALC se habría elaborado un modelo srilankés de desarrollo; y; si las elaboraciones realizadas teniendo como componentes a las ideas procedentes de ALC serían similares a las elaboradas en otros lugares del mundo.

\section{Elementos comunes en las reelaboraciones}

En los 3 autores estudiados existe una similitud decisiva, desde el punto de vista que interesa destacar y que ha sido el criterio básico para incluirlos en el estudio: la recepción de pensamiento proveniente de ALC y su consideración en las síntesis elaboradas posteriormente. Puede considerarse también un elemento común, al 


\section{Eduardo Devés-Valdés}

menos para los años 1960s y 1970s, la relativamente baja presencia de ingredientes eidéticos provenientes de la propia trayectoria intelectual de S-L. En ese momento se encuentran muy escasas alusiones a elementos de la trayectoria cultural del país o del Subcontinente Indio como escasas o nulas alusiones a factores económico-sociales autóctonos o ancestrales que sería necesario revivir o enactar, aunque en trabajos posteriores las haya más abundantes, particularmente en Goonatilake. Puede considerarse también como un elemento común, al menos a dos autores (Corea y Goonatilake) la cercanía al IDSSussex y es conocida la importancia de IDS como re-transmisor de ideas desde ALC a Asia y África, que ya ha sido destacada en otros textos (GOONATILAKE, 1975a; DEVÉS, 2004).

Dichas similitudes, sin embargo, no alcanzan para sostener que sobre la base de las ideas recibidas de ALC se habría constituido una escuela sirilankesa de pensamiento. Los autores no reciben suficientes ideas, esta recepción no se mantiene por suficiente tiempo, y tales ideas no se asumen con la importancia que se dio a otras como ha sido, por ejemplo el marxismo, incluso en su vertiente trotskista, en S-L.

\section{¿Modelo srilankés o asiático o tercermundista de desarrollo?}

Entonces ¿es o no posible hablar de un modelo de desarrollo que estos autores habrían formulado, inspirándose en los aportes provenientes de ALC?

Como construcción común o coincidente, no es posible. De hecho, no hay suficiente armonía o consenso entre los tres autores estudiados como para detectar un modelo de desarrollo que se habría inspirado en insumos venidos de ALC, combinados con otros de cualquier proveniencia, y ello aunque Corea y, particularmente, Ponnambalam intentan elaborar un modelo de desarrollo que responda a la realidad a la que se encuentran abocados: Corea, al amplio Tercer Mundo o al Sur, y Ponnambalam, específicamente a S-L.

No obstante, lo que es relevante es que ambos autores elaboran propuestas de desarrollo inspirándose inicialmente en ideas 
El impacto y la reelaboración de las ciencias económico-sociales...

elaboradas en ALC y tendiendo a llevarlas más allá: Corea lo intento desde la perspectiva de los organismos internacionales, desde la macropolítica del desarrollo y desde la propuesta de una reforma de la economía mundial, teniendo como principales agentes a los estados y, Ponnambalam, por su parte, mirando desde S-L, acentuó la auto-confianza de un pueblo que había sido desvalorizado por la actividad colonial y que, encontrando bloqueada su iniciativa histórica por esta causa, debía superarla sobre la base de la autoconfianza.

Ya se ha señalado, la propuesta de Corea podría ubicarse en la misma línea que la de Prebisch y en ALC fue seguida de manera muy similar por otro discípulo de Prebisch, el conductor de la CEPAL y del BID, Enrique Iglesias. La propuesta de Ponnambalam, en cambió, fue más original y no se elaboró simultánea o posteriormente por parte de autoras latinoamericanas, aunque en cierto modo fuera anticipada por el caribeño, residente e Tanzania, Walter Rodney a inicios de los 1970s.

\section{Comparaciones con otros autores}

Por otra parte, ¿es o no posible mostrar paralelos entre las maneras como los srilankeses trataron los insumos eidéticos provenientes de ALC y la manera como lo hicieron otros autores en Asia o África?

Sí, aunque sin pretender que la respuesta positiva sea válida para los tres autores en el mismo sentido. Siendo los 3 autores bastante diferentes, las similitudes con otros también lo serán. Lo más cercano, como ya se ha señalado es la similitud entre el Ponnambalam de Capitalismo dependiente en crisis y el Walter Rodney de Cómo Europa subdesarrolló Africa (1974). Al igual que Rodney, intenta pensar una región postcolonial, subdesarrollada y dependiente que no logra emerger de dicha situación. Para entender el por qué, como Rodney, se inspira en Fanon, en Nyerere y en Nkrumah, pero no alude, como lo hace el caribeño-tanzano, al panafricanismo, a la historiografía de la iniciativa africana ni a los ulemas argelinos. 


\section{Eduardo Devés-Valdés}

La posición de Corea, por su parte, podría equipararse a la de Jagdish Bhagwati $(1976 ; 1977)$, por su énfasis en el desarrollo asociado al sistema económico mundial y por su recuperación de la historia del pensamiento económico emergido en las periferias (1969) pero, más bien, sus planteamientos son similares a los del uruguayo Enrique Iglesias, especialmente en su época de secretario del Banco Interamericano de Desarrollo (1999; 2001), tanto por su incondicional inspiración en R. Prebisch, por su insistencia en la necesidad de un pacto Norte-Sur que mejore las condiciones de los países subdesarrollados y por el énfasis en el papel que les tocaría jugar a los organismos internacionales.

La posición del Goonatilake de 1976, con sus polémicas en el IDS Bulletin, sería parangonable a la del pakistaní Mahbub ul Haq, de comienzos de los 1970s, y sus textos respecto del Foro Tercer Mundo (1974; 1975), ocupado en la búsqueda de un discurso propio de la periferia. Goonatilake, como Ul-Haq, postula que el discurso del centro ha inhibido y/o silenciado los discursos de las periferias. El pakistaní postuló que la intelectualidad del Tercer Mundo había vivido de conceptos y pensamientos en gran parte tomados de Occidente y que su pensamiento ha sido frecuentemente juzgado con los estándares occidentales (1975, p. 9). Para él, la independencia nacional no es completa si la liberación política no es seguida de liberación económica e intelectual (1975, p. 10), y la liberación intelectual es la más difícil de alcanzar, dado que buena parte de la intelectualidad del Tercer Mundo permanece prisionera de su pasado educacional. Ul-Haq postula entonces la necesidad de hacer del Foro Tercer Mundo un movimiento de autosuficiencia (self-reliance) intelectual.

\section{Bueno ¿y entonces?}

Entonces debe reiterarse que las ciencias económico-sociales originadas en ALC tuvieron impacto, de variado alcance, en varios autores oriundos de S-L, que no alcanzaron a dar forma algo que 
El impacto y la reelaboración de las ciencias económico-sociales...

podría llamarse un "cepalino-dependentismo sirilankés", aunque se encuentran como componentes de diversos discursos contemporáneos presentes allí.

Mahbub ul Haq escribió: "Las ideas tienen una vida propia, y no hay nada más fascinante que observar su desarrollo" (1978). Estas ideas latinoamericanas, que salieron a vagar por el mundo, tuvieron variadas historias, por así decir, haciendo sus propias vidas lugares muy diversos. Allí se instalaron, se cruzaron y tuvieron descendencia criolla. Debieron cumplir tareas o funciones que no sospechaban, algunas de las cuales no sabían siquiera que existían.

La propia intelectualidad latinoamericana no ha acogido (no ha sido capaz de acoger) de vuelta estas ideas que echó al mundo, permaneciendo sin siquiera reconocerlas. Existe descendencia eidética latinoamericana no únicamente en S-L, sino en numerosos países, siguiendo en distintos lugares trayectorias diferentes. Esto hace que nuestro "patrimonio eidético" sea mayor que el que creemos y reconocemos y que acogerlo sea de gran interés para nosotros mismos, para fecundar nuestro propio pensamiento y para pensar más-mejor.

The impact and the re-elaboration of Latin-American economic-social sciences in Sri Lanka. Study about 3 authors: Gamani Corea, Satchi Ponnambalam and Susantha Goonatilake

Abstract. The economic-social sciences in the 1960s and the 1970s was the world-wide most recognized Latin- American and Caribbean eidetic production. Its vast circulation reached several regions, including not even thought places. Among the numerous African and Asian countries where it was recognized, we must mention Sri-Lanka. Considering the impact of those economic-social sciences in Sri Lanka, we studied the work of three authors: Gamani Corea, Susantha Goonatilake and Satchi Ponnambalam.Our intention was to give an answer to two questions:

1-What aspects of these economic-social sciences had the most ample reception? We concluded that among the authors, the most cited have been Raúl Prebisch y André G. Frank; in relation to language, the production in English was quite more receipted than the other ones; about the filter, the ideas most abundantly received were those previously digested by the Anglo-Saxon intellectual community working about development. 


\section{Eduardo Devés-Valdés}

2-What theoretical elaborations created Sri-Lankan authors, having those economic-social sciences as nourishment? The conclusions go in two levels: first, in relation with crossings made, we detect tendencies proceeding from the economy of development, from third-world ideologies and African socialism; and second, in relation with the characterization or classification of the intellectual products or new eidetic systems elaborated, we can talk about "thirdwoldist and identitarian development theories".

Keywords: Economic and Social sciences. Sri-Lanka. ECLA. Development. Dependence. Circulation of Ideas. Eidect Studies.

\section{Notas}

${ }^{1}$ Este artículo es producto del proyecto Fondecyt No 1070104 . Se ha realizado sobre la base de investigaciones llevadas a cabo en la Jawaharlal Nerhu University y en el Jawaharlal Nerhu Museum de Nueva Delhi, y agradezco particularmente al profesor Shyama Prasad Ganguly y al profesor Abdul Nafey; en CODESRIADakar, agradezco particularmente a la profesora Sokhna Gueye; en IDEP y Universidad Ch. A. Diop de Senegal, agradezco particularmente al profesor Amadou Ndoye; en el "Centro de Estudos Afro-Asiáticos" de la Universidad Candido Mendes de Río de Janeiro, agradezco particularmente al profesor Beluce Bellucci; en la Universidad de Puerto Rico en Río Piedras, en la biblioteca de la CEPAL en Santiago de Chile, a través de búsquedas en Internet y de la correspondencia con los propios autores estudiados, como Susantha Goonatilake, cuando ello ha sido posible.

${ }^{2}$ Muchos de los términos que corresponden a personas, escuelas de pensamiento, instituciones y redes, pueden consultarse en ATLAS DEL PENSAMIENTO en www.umbral.uprrp.edu Allí podrá encontrarse gran cantidad de informaciones y de conexiones acerca de estos asuntos. Asumiendo la necesidad de una producción industrial de artículos, se ha optado por la fórmula que consiste en subir a Internet una gran cantidad de piezas, llamémoslas "accesorias", de modo de no tener que incorporarlas a cada artículo, especialmente si van a ser publicados en papel. Incorporarlas cada vez implicaría un encarecimiento sustancial, por el crecimiento del texto. El proyecto ATLAS DEL PENSAMIENTO, como su nombre lo indica, apunta a exhibir geográficamente el pensamiento, particularmente de las regiones periféricas, durante los 3 últimos siglos. En el texto impreso se entregan únicamente las referencias más necesarias.

${ }^{3}$ La UNCTAD (United Nations Conference for Trade and Development) nació en 1963-4, principalmente para servir como institución que ayudara a los países en

Anos 90, Porto Alegre, v. 17, n. 32, p. 45-73, dez. 2010 
El impacto y la reelaboración de las ciencias económico-sociales...

vías de desarrollo a consolidar su posición y conducir sus negociaciones en iguales términos que los países industrializados. En vistas a este objetivo la UNCTAD construyó el mayor programa de investigación para analizar los problemas del desarrollo. Pero el propósito principal era construir la capacidad negociadora de los países en desarrollo en todos los foros internacionales (SENGUPTA, 2008). ${ }^{4}$ El propio Goonatilake (2009) reconoce, sin embargo, en carta al autor, que leyó muy poco a los autores de ALC. Mi percepción es que conoció mucho más lo que se decía, lo que vagamente se había difundido, que la obra específica de unos u otros. Es decir, lo conocía como un pensamiento que se manejaba en el medioambiente intelectual en el que se movía en Sussex, pero sin detalles ni diferenciaciones específicas.

${ }^{5}$ De hecho, por ejemplo, en Raúl Prebisch, que alude tan escasamente a su trayectoria eidética, es fácil detectar la presencia de ideas integracionistas, industrialistas, anti-imperialistas, proteccionistas, estatistas y otras de larga data en el medioambiente intelectual argentino y latinoamericano de 1950, cuando Prebisch comienza la elaboración de su nuevo "sistema eidético", el cepalino-clásico. Sabemos de sus conexiones con la trayectoria del socialismo argentino y con Juan B. Justo, de sus conexiones con Alejandro Bunge. Sabemos también de la presencia en ese medioambiente numerosas especies eidéticas, por señalar apenas unas pocas, como el pensamiento de Manuel Ugarte, el pensamiento de Víctor R. Haya de la Torre y un amplio aprismo, y las variopintas expresiones del nacionalismo argentino, algunas de las cuales constituyeron la base del peronismo. Todas estas tendencias eran ineludibles en ese medio intelectual, aunque Prebisch no aluda a ellas. Por otra parte, en unas u otras se manifestaba el pensamiento de List, Hobson, Lenin y Trotski, Estalin, del franquismo y del fascismo y sobre esas bases fue recepcionado el pensamiento de Mijail Manoilesco o de J. M. Keynes (DEVÉS, 2000; 2003).

${ }^{6}$ En verdad, la cantidad de ingredientes no hace necesariamente mejor el resultado, aunque es mucho más apetitoso para el gourmet que, en una suerte de degustación barroca, va a poder con las papilas del espíritu realizar múltiples interpretaciones de un mismo guiso: cada contraste, cada acentuación de sabores, cada mezcla o combinación, cada cruzamiento, será evaluado de manera independiente.

${ }^{7}$ Se denominan "motivos periféricos" a un conjunto de tópicos reiterados por las intelectualidades de diversas regiones de la periferia, aludiendo a las relaciones con el centro. Es particularmente importante el motivo que señala que el pensamiento-conocimiento del centro ha sido antecedido y/o se ha afirmado en el pensamiento-conocimiento elaborado previamente en las actuales periferias. Esto ha sido muy reiterado por las intelectualidades de India, China y de las regiones islámicas e incluso en ALC en algunos casos. Se ha insistido, por ejemplo, en que la astronomía maya era más elaborada y avanzada que la occidental.

Anos 90, Porto Alegre, v. 17, n. 32, p. 45-73, dez. 2010 


\section{Eduardo Devés-Valdés}

\section{Referências}

ASIRWATHAN, S. R. Les sciences sociales a Sri Lanka: politique, recherche et formation In: CHERNS, A. B. Les sciences sociales: organisation et politiques, Paris: UNESCO, 1975.

COREA, Gamani. A Message from the Secretary General of UNCTAD. In: IDS Bulletin, v. 7, n. 4, Sussex University, mayo, 1976.

COREA, Gamani. Need for change. Towards the new international economic order, Pergamon Press, Oxford-New Cork, 1981.

COREA, Gamani. Creating a framework to strengthen and stabilize international commodity markets, In: HENRIKSON, Alan (Edit.) Negotiating world order. The artisanship and architecture of global diplomacy, Scholarly Resources, Wilmington-Delaware, 1984a.

COREA, Gamani. Development and recovery: the realities of a new interdependence, United Nations, New York, 1984b.

COREA, Gamani. Global stakes require a new consensus. In: Development. Journal of SID, v. 3/4, 1990.

COREA, Gamani. My Memoirs, Published by the Gamani Corea Foundation, Vishva Lekha Printers, p. 483, 2008.

COREA, Gamani. 11 ${ }^{\text {th }}$ RAÚL PREBISCH LECTURE Geneva, 11 October 2001. Disponible en: www.unctad.org/en/docs/prebisch11th.en.pdf, 2001.

DESAI, Padma. Third World social scientist in Santiago. In: World Development, v. 1, n. 9, Septiembre, 1973.

DEVÉS, Eduardo. Las ideas económico-sociales de América Latina y el Caribe en Pakistán: recepción y reelaboración (1965-1980), enviado a revista Atenea, Universidad de Concepción, 2010.

DEVÉS, Eduardo. Recepción y reelaboración del pensamiento económico-social chileno y latinoamericano en Tanzania 1965-1985: Su proceso de africanización", Atenea, n. 492, Universidad de Concepción, 2005.

DEVÉS, Eduardo. ¿Cómo pasaron las ideas socioeconómicas Latinoamericanas a África anglófona entre 1960-1980? Retransmisores de ideas Latinoamericanas: Dudley Seers y el Institute of Development Studies. In: Latinoamérica. Revista de Estudios Latinoamericanos, CCYDEL-UNAM, México, 2004.

DEVÉS, Eduardo. El pensamiento latinoamericano en el siglo XX. Entre la modernización y la identidad, Tomo I: Del Ariel de Rodó a la CEPAL; Tomo II

Anos 90, Porto Alegre, v. 17, n. 32, p. 45-73, dez. 2010 
El impacto y la reelaboración de las ciencias económico-sociales...

Desde la CEPAL al neoliberalismo; Tomo III Las figuras y las ideas del fin de siglo, Biblos. Buenos Aires: DIBAM, Santiago, 2000-2003.

DEVÉS, Eduardo; ROSS, Cesar. Las ciencias económico-sociales latinoamericanas en Africa Sudsabariana, Ariadna, Santiago, 2009.

FRANK, André G. El subdesarrollo del desarrollo. Un ensayo autobiográfico, Caracas: Nueva Sociedad, 1991.

GOONATILAKE, Susantha. Development thinking as cultural neo-colonialism - the case of Sri Lanka. In: IDS Bulletin, v. 7, n. 1, Sussex University, 1975a.

GOONATILAKE, Susantha. Cultural dependence. The debate continues. In: IDS Bulletin, v. 7, n. 3, Sussex University, 1975b.

GOONATILAKE, Susantha. Aborted discovery. Science and creativity in the Third World, Londres: Zed-Press, 1984.

GOONATILAKE, Susantha. Modern science and the periphery. The characteristics on the dependent knowledge. In: HARDING, Sandra (Edit.) The "racial" economy of science. Towars a democratic future, Bloomington: Indiana U. Press, 1993.

GOONATILAKE, Susantha. A monk for and of our time. Disponible en: www. sundaytimes.lk/001112/news4.html. Acesso em 12 de nov. 2000.

GOONATILAKE, Susantha. Coming Intellectual Shifts to Asia: The Indic Possibilities. Disponible en: www.infinityfoundation.com/indic_colloq/.../ paper_goonatilake.pdf, 2002.

GOONATILAKE, Susantha. Carta a Eduardo Devés. In: archivo del autor, 2009.

IGLESIAS, Enrique. Raúl Prebisch tenía razón. En el centenario de su nacimiento, las ideas del legendario economista argentino, creador de la CEPAL, recobran vigencia. Disponible en: www.caretas.com.pe/2001/1686/articulos/prebisch. phtml, 2001.

IGLESIAS, Enrique. Cambio y crecimiento en América Latina 1988-1998 Ideas y acciones, Washington DC: BID, 1999.

JUPP, James. Democratic socialism in Sri Lanka. In: Pacific Affairs, v. 50, n. 4, University of British Columbia Stable URL, winter 1978.

LERSKI, Geoge Jan. Origins of Trotskiyism in Ceylon, Standford: Stanford U. Press, 1968.

LEWIS, Rupert. Walter Rodney's intellectual and political thought. The Press University of the West Indies, Barbados, Jamaica, Trinidad-Tobago, 1998.

PIERIS, Aloysius. An Asian theology of liberation, New York: Orbis Books, 1988.

Anos 90, Porto Alegre, v. 17, n. 32, p. 45-73, dez. 2010 


\section{Eduardo Devés-Valdés}

PREBISCH, Raúl. Cinco etapas de mi pensamiento. In: El Trimestre Económico, n. 198, junio, 1983.

PONNAMBALAM, Satchi. Dependent capitalism in crisis. The Sri Lankan economy, 1948-1980, Londres: Zed-Press, 1981.

PONNAMBALAM, Satchi. Sri Lanka: National Conflict and the Tamil Liberation Struggle, Londres: Zed Books, 1983.

PONNAMBALAM, Satchi. Tamil Liberation Struggle Reaches Popular Stage. Proceedings of the Second World Tamil Convention, Nanuet, New York, U.S.A. Disponible en: www.tamilcanadian.com/page.php?cat=27\&id=53, 1984.

ROBERTS, Michael. For humanity. For the Sinhalese. Dharmapala as crusading Bosat. The Journal of Asian Studies; v. 56, n. 4, nov 1997.

RODNEY, Walter. How Europe underdeveloped Africa, Washington D.C.: Howard University Press, 1974 [1 $1^{\circ}$ edic. 1972].

SANKATSING, Glenn. Development and society in the Americas. Disponible en: www.crscenter.com, 2007.

SENGUPTA, Arjun. Gamani Corea, and the North-South Relations. Disponible en: http://www.island.lk/2008/09/14/features3.html, 2008.

STAVENHAGEN, Rodolfo. Decolonialising Applied Social Sciences, en Human Organization, v. 30, n. 4, 1971.

UL HAQ, Mahbub. Development and Independence In: Development Dialogue, n. 1,1974 .

UL HAQ, Mahbub. The Third World Forum: Intellectual Self-Reliance In: International Development Review, n. 1, London, 1975.

UL HAQ, Mahbub. La cortina de la pobreza, F.C.E., México, 1978.

Recebido em 01/12/2010

Aprovado em 30/01/2011 